\title{
Tensor-based tag emotion aware recommendation with probabilistic ranking
}

\author{
Hyewon Lim* and Hyoung-Joo Kim \\ School of Computer Science and Engineering, Seoul National University \\ Republic of Korea \\ [e-mail: hwlim@idb.snu.ac.kr, hjk@snu.ac.kr] \\ *Corresponding author: Hyewon Lim
}

Received 10 August, 2018; revised October 31, 2018; revised March 18, 2019; accepted August 3, 2019; published December 31, 2019

\begin{abstract}
In our previous research, we proposed a tag emotion-based item recommendation scheme. The ternary associations among users, items, and tags are described as a three-order tensor in order to capture the emotions in tags. The candidates for recommendation are created based on the latent semantics derived by a high-order singular value decomposition technique (HOSVD). However, the tensor is very sparse because the number of tagged items is smaller than the amount of all items. The previous research do not consider the previous behaviors of users and items. To mitigate the problems, in this paper, the item-based collaborative filtering scheme is used to build an extended data. We also apply the probabilistic ranking algorithm considering the user and item profiles to improve the recommendation performance. The proposed method is evaluated based on Movielens dataset, and the results show that our approach improves the performance compared to other methods.
\end{abstract}

Keywords: Recommendation, tag, High-Order Singular Value Decomposition (HOSVD), BM25, item-based filtering

This work was supported by Institute for Information \& communications Technology Promotion (IITP) grant funded by the Korea government (MSIP) (No.R0113-15-0005, Development of an Unified Data Engineering Technology for Largescale Transaction Processing and Real-time Complex Analytics) 


\section{Introduction}

Social cataloging services allow users to catalog items, tag and rate them, write reviews, and join groups. The aim of the services is expressing opinions and sharing ideas as well as the easy information retrieval [1]. The social cataloging service furnishes the item's metadata for the user's convenience. However, users still spend much time searching items for them; it aggravates the fatigue of item selection.

In many social communities including social cataloging services, providing better recommendation is an important issue. The recommender system plays a role as a filter for overloading information. The user behaviors in the service can be assumed as private preferences and interests [2]-[4]. Collaborative filtering and content-based method are two primary recommendation approaches. The collaborative filtering is to predict new items the user may like considering other users who have similar interests with the target user. The content-based filtering is to recommend new items similar to the user's previous item selection.

The recommender systems should improve users' satisfaction by providing the appropriate information relying on personal need, it is necessary to take into account the user's feedback as well as the item information. Recently, in order to improve the user's satisfaction with the recommendation, many researchers analyze the emotions of users included in the subjective feedback and use them as the feature of the recommendation. The user's emotion play a significant part in selecting and consuming items. User's decisions are made with their feelings, and user's demands are forgotten when their emotional needs are gratified [5]. According to [6], emotions aroused from an action before consuming an item affect the user's next selection, and emotions after the consumption affect the user's next behavior. The post-consumption feeling can be very helpful in estimating the satisfaction of the user with the item. In the social cataloging system, the user's behaviors such as rating and tagging can be presumed as the actions reflected the post emotion.

Tags, which is a keyword annotated to items freely by users, help users to catalog and retrieve items. In addition, tags are regarded as a user's implicit rating or voting for tagged items [7] and serve to expose personal preference. For these reasons, tags are frequently used as the feature of the recommendation. Even if the same tag is used for different items, the meaning of the tag can be either positive or negative. Therefore, considering the user's intention in the tag is needed for better understanding of the user's preference.

In the conventional recommender systems, the relationships among users, items, and tags are modeled by a tripartite graph. In this case, the ternary relationship is transformed into three binary relationships, i.e., users-items, items-tags, and users-tags, and it is difficult to consider total information among the triplets [8]. Tensor modeling is well-known method for solving this problem. The user's tag assignment can be modeled as a tensor, which is an n-dimensional array, and the latent relationships can be derived using the tensor factorization [8], [9].

In our previous research [10], we proposed a recommendation method that uses the tenser modeling scheme to consider the emotions in the tag. However, this approach has two limitations. First, since only a few items are tagged by users and the user-provided tag are various, the tensor is highly sparse. Second, after the process of the tensor factorization and reconstruction, the value in the tensor can be assumed as the preference of the user for the item, and the items are ranked using the value [8], [11], [12]. However, these approaches do not 
consider the user's past activities [9]. In this paper, we suggest a recommendation method for mitigating two limitations and improving the efficiency of the recommendation based on our previous study. In order to reduce the tensor sparsity, we generate new data entries using collaborative filtering and combine them with the original dataset to make a larger dataset. For improving the recommendation performance, we use BM25 weighting scheme [13] to create user and item profile based on their tags. The experimental evaluations for measuring the performance of our research are conducted using the Movielens dataset. The experiments show that adding new entries based on user data and ranking candidates based on user and item profiles improve the performance of recommendation.

The organization of the rest of this paper is as follows: related work is showed in Section 2. In Section 3, we explain the recommendation approach proposed in our previous study, and introduce item addition method and candidate ranking method in Section 4. The experimental evaluations of the proposed method are provided in Section 5. Section 6 discusses the conclusion and future research.

\section{Related Work}

Collaborative filtering and content-based filtering are typical recommendation approaches. This approach recommends new items based on the preferences of other users who have similar interests with the target user. The information of items is not considered, but ratings. In contrast, content-based filtering is to focus on the items' features and recommend items based on the user's previous item list. Those recommendation methods have various problems such as data sparsity, coldstart problem, grey-sheep problem, and scalability. Hybrid recommendation techniques which is the combination of two approaches have suggested to avoid those limitations.

Much research have paid attention to the user's subjective feedback as well as the features of items to improve the user's satisfaction with the recommendation. Among the various feedbacks, many studies have focused on tags [14]-[17] because tag is used not only to facilitate item retrieval, but to represent the user's opinions. [14]. Guy et al. [18] suggested an item recommendation technique which combines the information of users and tags. They create the user's profile based on the diverse relations between user and tag. Zhang et al. [19] considered the two roles of the tags which are the item organization and the connection between user and item. They argue that the tags' role as the connection is very helpful to recommendation; the hybrid approach shows the best result. In the study of [20], users are modeled based on their tags. The items are classified into two sets by the polarity and found the relevant topics using the tags. To improve the quality of the recommendation, Gedikli et al. [21], [22] have studied to predict the item's rating by generating rating on the tag itself. For item recommendation, Kim et al. [23] used the implicit trust relationships derived from the tagging behaviors of the user. Huang et al. [24] proposed the FRD model combining the tags' frequency, recency and duration to find the personal preferences of the user. They applied collaborative filtering and content-based filtering to find the similar users and items to the target user. The recommendation score is computed based on the target user's FRD information.

The ternary relationships among users, items, and tags are modeled by a tripartite graph. In the graph, three pairs of the relationships, i.e., user-item, user-tag, and item-tag, can be captured, but co-existence information about three elements can be lost [11]. The recommendation quality of modeling the ternary relationship with a multidimensional matrix 
is better than the one with a tripartite graph because the ternary relationship can be considered at once. Researchers have used a three-order tensor to model the ternary association and apply the tensor factorization to capture the latent semantics. HOSVD [25], which is one of the tensor factorization methods, has been applied in diverse research. Symeonidis et al. [8], [26] have suggested a tensor-based recommendation method. A three-order tensor for the ternary relationships was used and applied the HOSVD technique on the tenser, and a Kernel-SVD combination technique was adopted to improve the recommendation accuracy. Peng et al. [11] introduced the concept of hidden tag and hidden item to grasp the similarity between users and Tucker decomposition was used to the recommendation. Xu et al. [27] applied HOSVD technique to improve personalized web search. They split a tensor into several sub-tensors for mitigating the sparsity problem. In the study of [9], the ranking scheme of the recommendation cantidates and the scalability in the tensor reconstruction are investigated. They argued that the result of the tensor reconstruction disregards the user's past behaviors. Therefore, they suggested that the result of tensor modeling ranked by applying Naïve Bayes. To reduce the tensor computation size, the research of Rafailidis et al. [28] suggested the tag clustering using K-means algorithm and tripartite clustering method. The research of [29]-[31] have improved the traditional matrix factorization techniques to be more robust to noise and outliers. The authors have used the deep learning to obtain the rich information about images and to reduce the semantic gap between images and tags.

In order to improve the efficiency of recommender systems, many studies have focused on users' emotional information [5], [32]. SenticRank [33] is the framework which maps the user profile derived by tags to the sentiment space and finds the proper resources to the user's query. The research was for personal search, but the framework can be applied to the recommendation. Qingbiao et al. [34] proposed a sentiment enhanced recommendation method which utilizes the polarity of tag synsets to calculate resources' similarities. Dong et al. [35] combined the product similarity and sentiment. The sentiment of the product is generated by features of the user's review. Sun et al. [36] and Garcia et al. [37] and have applied the sentiment analysis on the feedback of the user. Both studies reported that the sentiment analysis is effective to improve the quality of the recommendation.

\section{Tensor-based Item Recommendation with Tag Emotion}

Users in social cataloging service use tags to express their impression and interest in the item. A tag is considered as an element for the personalized recommendation because it provides not only an additional information about the item, but also a subjective feedback of the user who tagged the item. The tag-based recommendation techniques represent the relationships among users, items, and tags as the three pairs of relationships: user-item, user-tag, and item-tag. In this case, the co-existence information existing among the triplets is lost [11]. To preserve and apply the information to the recommendation, the ternary relationships are rep resented as a multi-dimensional matrix instead of a tripartite graph.

In this section, we briefly describe our previous research that the recommendation method considering the tag emotions using tensor modeling [10]. The ternary relationships are constructed as a three-order tensor, and tag emotion is calculated based on the user's ratings and the emotion of each tag. To recommend new items, HOSVD is applied to tensor. We also describe an approach for combining new data to dataset using item-based collaborative filtering to reduce sparsity and candidate ranking method based on BM25 weighting scheme. An overview of the proposed recommendation method is illustrated in Fig. 1. 


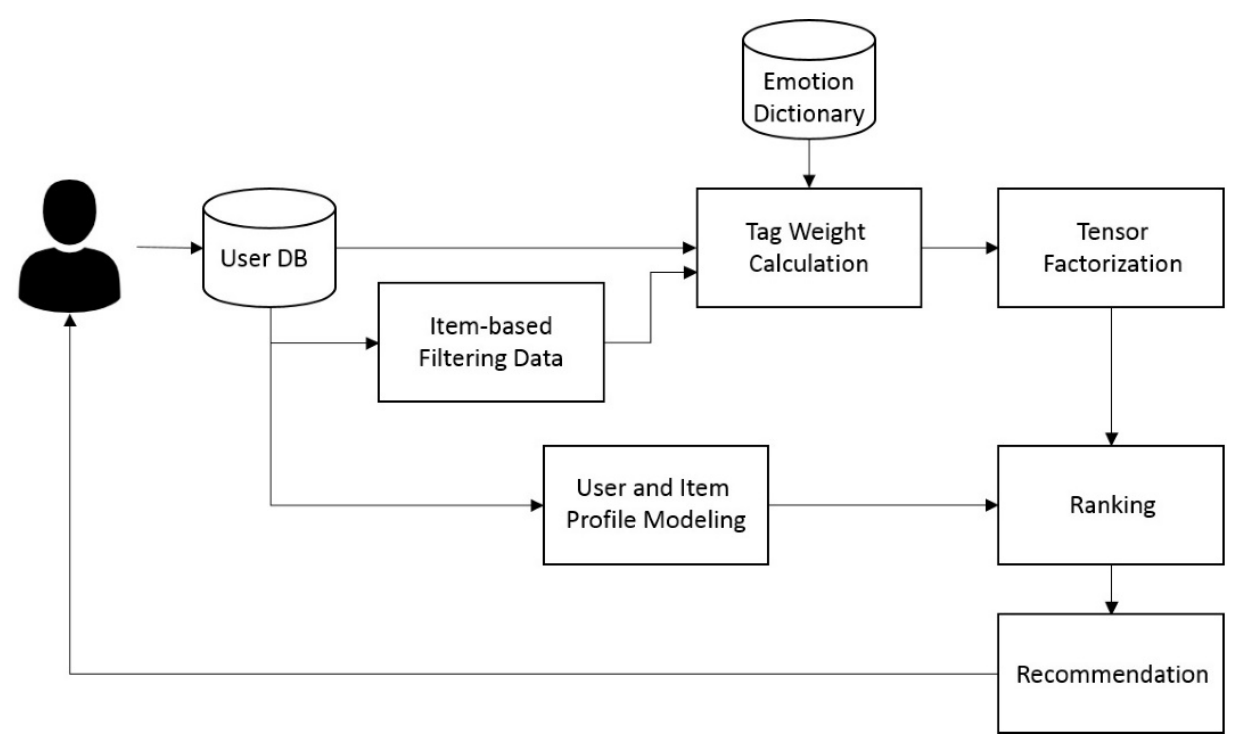

Fig. 1. Overview of the proposed scheme

\subsection{HOSVD}

Tensor factorization is a recommendation technique for handling multi-dimensional data. HOSVD [25] is a technique for applying SVD to a tensor. The input data is represented by a tensor $\mathcal{A}$, and the output is a reconstructed data $\hat{\mathcal{A}}$ which contains the likeliness of a user $u$ assigning a tag $t$ to an item $i$. The user receives an item recommendation based on the value of $\hat{\mathcal{A}}$. Suppose that three users tagged three movie items: $u_{1}$ assigns $t_{1}$ to $m_{3}$ and $t_{3}$ to $m_{2}, u_{2}$ assigns $t_{2}$ to $m_{1}$, and $u_{3}$ assigns $t_{3}$ to $m_{2}$. A three-order tensor $\in \mathcal{R}^{3 \times 3 \times 3}$ is generated for the usage data and the possible value of each element of the tensor is the likeliness of the ternary relationship as described in Table $\mathbf{1 .}$

Table 1. A usage data

\begin{tabular}{|c|c|c|c|}
\hline User & Movie & Tag & Weight \\
\hline \hline 1 & 2 & 3 & 1 \\
\hline 1 & 3 & 1 & 1 \\
\hline 2 & 1 & 2 & 1 \\
\hline 3 & 2 & 3 & 1 \\
\hline
\end{tabular}

To derive the latent semantics among the users, the items, and the tags, the tensor factorization is applied to the initial tensor $\mathcal{A}$. The three-order tensor is transformed into two-dimensional matrices through the unfolding process, and the core tensor $\mathcal{S}$ is constructed using the $n$-mode singular vectors obtained by applying SVD to the matrices. The core tensor manages the information of interaction among ternary relationships. The parameter $c_{j}$ is number of the dimensions to truncate for $j$-mode for filtering out the small singular values.

$$
\mathcal{S}=\mathcal{A} \times{ }_{1} U_{c 1}^{(1)^{T}} \times_{2} U_{c 2}^{(2)^{T}} \times_{3} U_{c 3}^{(3)^{T}}
$$


The core tensor $\mathrm{S}$ and the three factor matrices are applied $j$-mode product to obtain the reconstructed tensor $\hat{\mathcal{A}}$. Table 2 shows the reconstructed tensor of the usage data after applying HOSVD. The new entries become the candidates for recommendation.

$$
\hat{\mathcal{A}}=\mathcal{S} \times{ }_{1} U_{c 1}^{(1)} \times_{2} U_{c 2}^{(2)} \times_{3} U_{c 3}^{(3)}
$$

Table 2. A reconstructed tensor $\widehat{\mathcal{A}}$; new entry is generated.

\begin{tabular}{|c|c|c|c|}
\hline User & Movie & Tag & Weight \\
\hline \hline 1 & 2 & 3 & 1.17 \\
\hline 1 & 3 & 1 & 0.72 \\
\hline 2 & 1 & 2 & 1 \\
\hline 3 & 2 & 3 & 0.72 \\
\hline 3 & $\mathbf{3}$ & $\mathbf{1}$ & $\mathbf{0 . 4 5}$ \\
\hline
\end{tabular}

\subsection{Emotion based Weighting Scheme}

The user's emotion after consuming an item affect the user's next behavior [6]; therefore, the emotion can be the key in measuring the user's satisfaction with the item. The user expresses his/her gratification with the item through the rating and describe more detailed and various intensity of polarity using the tags; therefore, the user's ratings and tags can be viewed as the feature that reflects the post-consumption emotion. In our previous study [10], we proposed a tag weighting scheme reflecting the user's preference for improving the recommendation performance. The user's feedback is used instead of the likeliness as the weight.

First, the ratings become the base weight of the tags because it is the result of condensing the user's impression and indicates the user's preference explicitly. However, if the original rating is used as it is, the bias can be occurred since the rating's range can be different depending on the users. Thus, the user's ratings are vectorized and normalized to a unit vector. The rating based tag weight for an item $i$ by a user $u$ is calculated as follows:

$$
\begin{aligned}
\text { weight }_{\text {base }}\left(t_{u, i}\right) & =\left\{\begin{array}{c}
r_{u, i}(t), \text { if } r_{u, i} \neq \emptyset \\
0, \text { otherwise }
\end{array}\right. \\
r_{u, i}(t) & =\frac{r_{u, i}}{\sqrt{\sum_{i=1}^{|I|} r_{u, i}^{2}}}
\end{aligned}
$$

where $r_{u, i}$ is the rating, and $r_{u, i}(t)$ is the normalized rating value.

Next, the emotion value of each tag is measured. The special characters included in tag and the proper nouns such as a name of person or a shop name are removed. If the proper noun contains the emotion word, the tag can be classified as an emotion tag even though it does not express emotion. To calculate the tag emotion based weight of each tag, the emotion dictionary is used. If a tag is present in the emotion dictionary, the emotion value is applied as the tag weight.

$$
\text { weight }_{\text {emotion }}\left(t_{u, i}\right)=\text { EmotionScore }\left(t_{u, i}\right)
$$

where EmotionScore $(t)$ is the emotion value of tag $t_{u, i}$. If the tag does not exist in the emotion dictionary, the value is 0 . 
If the tag is not in the emotion dictionary and comprises more than two words, the tag is splitted into words and the sum of the emotion values of each word is used as the weight of the

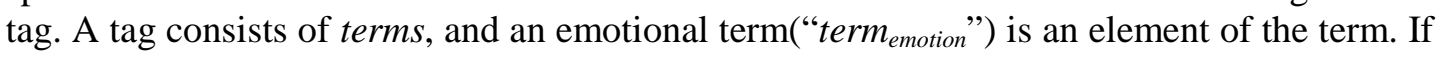
there is no emotion value, the tag weight becomes 0 .

$$
\text { weight }_{\text {emotion }}\left(t_{u, i}\right)=\frac{1}{\mid \text { term }_{\text {emotion }} \mid} \sum_{j=1}^{\mid \text {term } \mid} \operatorname{EmotionScore}\left(\text { term }_{j}\right)
$$

Finally, the total tag weight is calculated by combining each tag-based weight and the rating-based weight. If an item has multiple tags, the average of the tag weights becomes each tag's weight.

$$
\text { weight }\left(t_{u, i}\right)=(1-\alpha) \times \text { weight }_{\text {base }}\left(t_{u, i}\right)+\alpha \times\left(\text { weight }_{\text {emotion }}\left(t_{u, i}\right) \times 0.5\right)
$$

The influence of the emotion tag can be controlled by the parameter $\alpha$. The rating-based weight accounts for a large part of overall tag weight because the rating indicates the user's impression of an item explicitly. The $\alpha$ is set to 0.2 empirically. If an item is tagged with several tags by the user, each tag has the average weight of the tags.

\section{Improving Recommendation Accuracy}

\subsection{Generating additional data}

Sparsity is a common problem of the recommendation based on the user's previous behaviors [3]. This is because the users feedback only a few items, which is less than the number of the entire items. In order to reduce the data sparsity problem, we generate an additional data applying item-based collaborative filtering [38]. The item-based filtering utilizes users' ratings to measure the similarity between items and calculates the similar items' weighted average of ratings to predict the rating of the target item.

The items are divided into two groups. One is the items with the target user's tags and the other is the items with other users' tags. Based on the similarity of two groups, the most similar $k$ items with the target user's tagged items is found. In order to compute the similarity, the adjusted cosine similarity is applied to consider the difference in the rating scale of each user.

$$
\operatorname{sim}\left(i_{a}, i_{b}\right)=\frac{\sum_{u \in U}\left(r_{u, i_{a}}-\bar{r}_{u}\right) \cdot\left(r_{u, i_{b}}-\bar{r}_{u}\right)}{\sqrt{\sum_{u \in U}\left(r_{u, i_{a}}-\bar{r}_{u}\right)^{2}} \cdot \sqrt{\sum_{u \in U}\left(r_{u, i_{b}}-\bar{r}_{u}\right)^{2}}}
$$

where $r_{u, i_{a}}$ and $r_{u, i_{b}}$ are the ratings of the item $i_{a}$ and $i_{b}$ by $u . \overline{r_{u}}$ is the average rating of $u$.

For calculating the tag weights as described in Section 3, each item's rating is required; therefore, we predict the ratings for the $k$ similar items. The weighted average ratings of the $k$ items for the predicted rating of the target item is calculated as follows:

$$
r_{u, i}=\frac{\sum_{j \in I} \operatorname{sim}(i, j) \cdot r_{u, j}}{\sum_{i \in I} \operatorname{sim}(i, j)}
$$

To reduce the sparsity of the tensor, the additional data is combined with the original data. 


\subsection{BM25 based Candidate Ranking}

In general, the results of the tensor factorization and reconstruction becomes the recommendation candidates; they are sorted by the value in the reconstructed tensor. Then, the top $k$ items among the candidates are recommended to the user. However, the research of Ifada et al. [9] argued that the previous tagging behaviors of the users are not considered in this approach. The fundamental idea under the latent factor model is that the item selection of a user is controlled by a few features [39], while the preferences of the user are explained by characterizing the user profiles and user's item consuming patterns such as gender, actors, or genres. In this paper, we adopted Okapi BM25 [13] to generate the user and item profile based on the tags. The BM25 algorithm is a ranking model for document retrieval based on TF-IDF by calculating the relevance between a query and a document; it considers the document's length. The score between a query $Q$ and a document $D$ is calculated as follows:

$$
\operatorname{score}(Q, D)=\sum_{\text {term } \in Q} \log \frac{N}{d f_{\text {term }}} \cdot \frac{t f_{\text {term }, d} \cdot\left(k_{1}+1\right)}{t f_{\text {term }, d}+k_{1} \cdot\left(1-b \cdot \frac{L_{d}}{L_{\text {avg }}}\right)}
$$

where $L_{d}$ is the length of $d$ and $L_{\text {avg }}$ is the average length of the entire documents. If $k_{1}$ is large, the term frequency's weight is increased, the closer $b$ is to 1 , and the more weight is placed on the document's length. The standard values of $k_{1}$ and $b$ are 2 and 0.75 , respectively.

In the research of [40] and [41], BM25 applied to the folksonomy for improving the personalized search performance. We adopted the BM25 to generate user profiles and item profiles. When user $u_{l}$ tags item $i_{m}$ with tag $t_{p}$, the user profile based on the previous tagging behavior is computed as follows:

$$
\operatorname{score}\left(u_{l}, t_{p}\right)=\operatorname{iuf}\left(t_{p}\right) \cdot \frac{t f\left(u_{l}, t_{p}\right) \cdot\left(k_{1}+1\right)}{t f\left(u_{l}, t_{p}\right)+k_{1} \cdot\left(1-b \cdot \frac{N_{u_{l}, t}}{\operatorname{avg}\left(N_{u, t}\right)}\right)}
$$

where $t f\left(u_{l}, t_{p}\right)$ is the tag frequency, and $\operatorname{iuf}\left(t_{p}\right)$ is the user-based inversed tag frequency. $N_{u_{l}, t}$ is the total number of the tagging of $u_{l}$, and $\operatorname{avg}\left(N_{u, t}\right)$ is the average number of the tagging of the entire users. The user-based tag frequency and the inverse tag frequency are calculated as follows:

$$
\begin{gathered}
t f\left(u_{l}, t_{p}\right)=\frac{N_{u_{l}, t_{p}}}{N_{u_{l}, t}} \\
\operatorname{iuf}\left(t_{p}\right)=\log \frac{|U|}{N_{u, t_{p}}}
\end{gathered}
$$

The tag-based item profile based is calculated as follows:

$$
\operatorname{score}\left(i_{m}, t_{p}\right)=\operatorname{iif}\left(t_{p}\right) \cdot \frac{t f\left(i_{m}, t_{p}\right) \cdot\left(k_{1}+1\right)}{t f\left(i_{m}, t_{p}\right)+k_{1} \cdot\left(1-b \cdot \frac{N_{i_{m}, t}}{\operatorname{avg}\left(N_{i, t}\right)}\right)}
$$

where $t f\left(i_{m}, t_{p}\right)$ is the item-based tag frequency, and $\operatorname{iif}\left(t_{p}\right)$ is the inversed tag frequency. $N_{i_{m}, t}$ is the number of tags assigned to $i_{m}$, and $\operatorname{avg}\left(N_{i, t}\right)$ is the average number of the tags assigned to the entire items. The item-based tag frequency and the inversed tag frequency are calculated as follows: 


$$
\begin{aligned}
& t f\left(i_{m}, t_{p}\right)=\frac{N_{i_{m}, t_{p}}}{N_{i_{m}, t}} \\
& \operatorname{iif}\left(t_{p}\right)=\log \frac{|I|}{N_{i, t_{p}}}
\end{aligned}
$$

The user and item profile are generated based on the initial tensor. The result of the reconstructed tensor are ranked using the similarity of the profiles. The similarity of the user and the item profiles is computed using the BM25-based cosine similarity suggested by [41].

$$
\operatorname{sim}\left(u_{l}, i_{c}\right)=\frac{\sum_{t \in T}\left(\operatorname{score}\left(u_{l}, t_{p}\right) \cdot \operatorname{score}\left(i_{c}, t_{p}\right)\right)}{\sqrt{\sum_{t \in T}\left(\operatorname{score}\left(u_{l}, t_{p}\right)\right)^{2}} \cdot \sqrt{\sum_{t \in T}\left(\operatorname{score}\left(i_{c}, t_{p}\right)\right)^{2}}}
$$

where $i_{c}$ is the candidate item in the reconstructed tensor.

\section{Experimental Evaluation}

\subsection{Dataset}

We use the dataset of Movielens ${ }^{1}$ to evaluate the performance of the proposed approach. The dataset has 71,567 users, 10,681 movies, 10,000,054 rating history, and 95,580 tagging histories. There are 15,230 distinct tags, and 4,009 users use tags at least once. On average, each user rates 143 movies and has 10 distinct tags. Among the users who have tagging history, $40 \%$ of the users use only one tag.

We applied $p$-core [42] at level $k$ to the dataset to obtain the dense data by removing less frequently used tags and unfamiliar items. We set $k$ to 5 , and finally obtained 210 users, 544 movies, and 365 tags. For the experiments, we adopted 5-fold cross validation. For each fold, we utilized $80 \%$ of each user's previous behaviors as the training set and the rest $20 \%$ as the test set.

SenticNet 4.0 [43] was used as the emotion dictionary. To reduce the dimension of the tensor factorization, we preserved $80 \%$ of the diagonal matrix.

\subsection{Data addition}

The experimental evaluation to obtain the proper number of the similar movies for user-tag pair is conducted. The performance of recommending top $n$ movies was evaluated by changing the number of similar items, $k$. The results are shown in Fig. 2; each figure illustrates the precision, recall, and f1-score based on the change in $k$. The $x$-axis shows the number of recommended movies; the $y$-axis shows the precision, recall, and f1-score, respectively. The results indicate that the smaller the $k$ value, the more similar movies are selected. As the value of $k$ increases, a movie that is similar with some movies in the group is selected; the data become diverse. Therefore, when the value of $\mathrm{k}$ is small (large), recommending a small (large) number of movies tends to perform better. Exceptionally, when the most similar movie with the user's taste is added, the recommendation performance is improved irrespective of the number of the recommended movies. Therefore, we set $k$ to 1 for the next experiment.

\footnotetext{
${ }^{1}$ https://grouplens.org/datasets/movielens/
} 

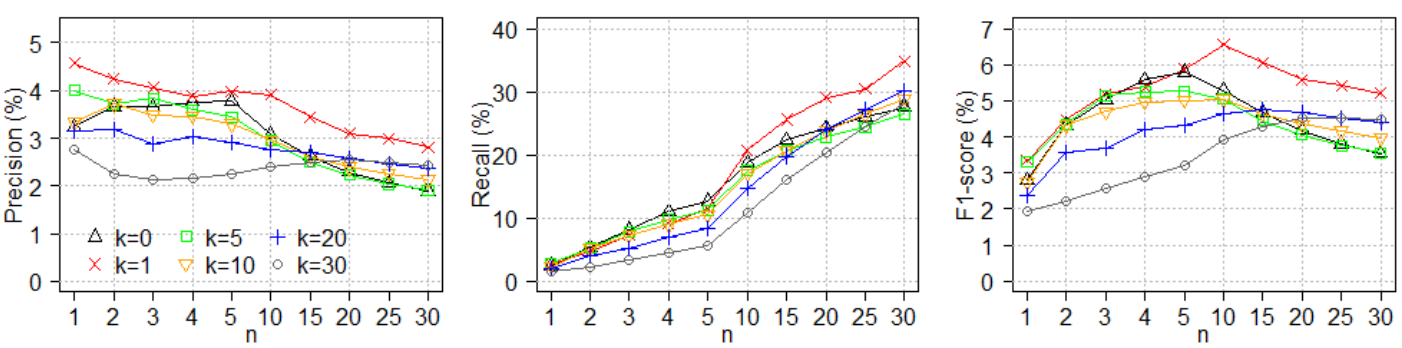

Fig. 2. The comparisons of precision, recall, and f1-score as the additional data increases.

\subsection{Recommendation Performances}

The performance of our proposed approach was compared with the following approaches: item-based collaborative filtering (CF) [38], the similarity fusion (SF) [44] which combines user-based collaborative filtering and item-based collaborative filtering by extending user-item matrix with tags, the standard tensor-based recommendation (TF) [8] that the likeliness of the ternary relationship is used as the tensor's weight, the tensor-based recommendation using Naïve Bayes (Bayes) [9], and SenticRank [33] which includes the sentiment in tag-based user profile.
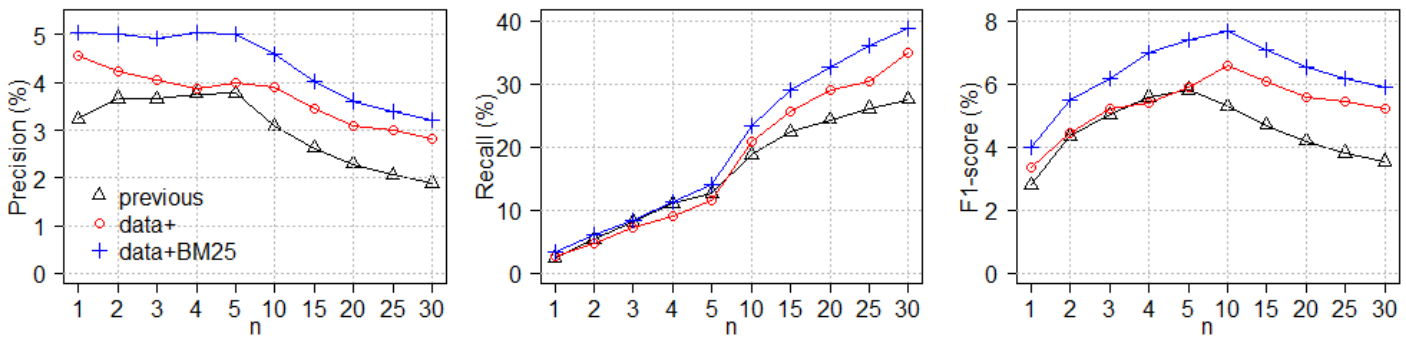

Fig. 3. The comparisons of precision, recall, and f1-score for the our approaches.
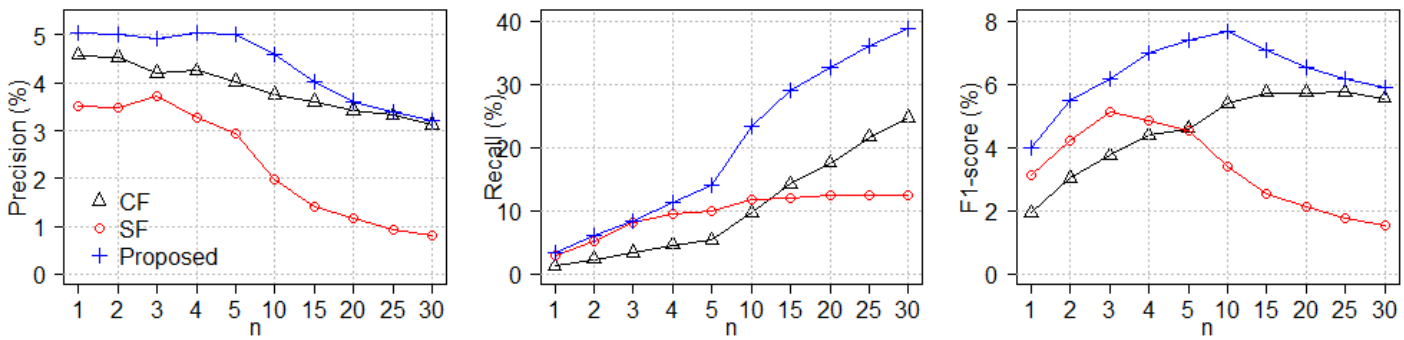

Fig. 4. The comparisons of precision, recall, and f1-score among the our approach, SF, and CF.

The top $n$ movies are predicted for a user, and the performances of the methods are measured by the precision, recall, and f1-score. First, we evaluate our approach's recommendation performance step by step: our previous research using original data and without the probabilistic ranking ("previous") [10], the approach with the data expansion 
("data+"), and the method with both the data addition and the ranking using BM25 (“data+BM25"). The results are depicted in Fig. 3: the precision, recall, and f1-score, respectively. The $x$-axis of shows the number of top $n$ movies; the $y$-axis shows the values of precision, recall, and f1-score, respectively. The results describe that using expanded data and profile-based ranking gives better performance than our previous study. It indicates that the recommendation performance can be improved by complementing the problems of lack of data or ignoring user behavior, which is considered to be the weak points of our previous research. Generating additional data using item-based filtering techniques serves as a supplement to the user experience that occupies very little part of the overall item, helping to make a richer recommendation while preserving the trend of user preferences. In addition, using tag-based user and item profile in the recommendation process enables a more personalized recommendation by filtering through items similar to the user's actual taste after identifying the user preference based on the latent semantic.

Next, we examine that the considering ternary relationships are better than the three pairs of relationship for recommendation, and the results are illustrated in Fig. 4. The comparison with CF considering only user-item pairs confirms that the user's tags are worth considering to improve the recommendation performance. It can be assumed that the tag plays a role in revealing the user's experience on the item more than the rating, and is in line with the previous studies emphasizing the importance of using tags. In the comparison between the proposed method and SF, the proposed method shows better performance than SF. However, the performance of the similarity fusion technique depends on the number of items the user has. In our experiment, only the top $1-5$ results are meaningful because the data is not sufficient. Nevertheless, given the fact that the performance of SF decreased after the top 3 in the f1-score result, considering the ternary relationships can prevent the information loss and improve the recommendation quality.
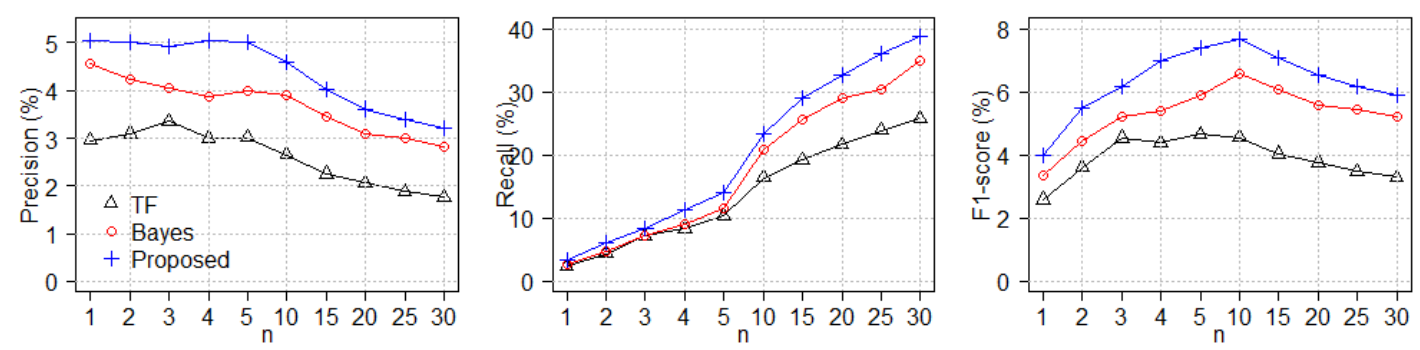

Fig. 5. The comparisons of precision, recall, and f1-score among the our approach, TF, and Bayes.
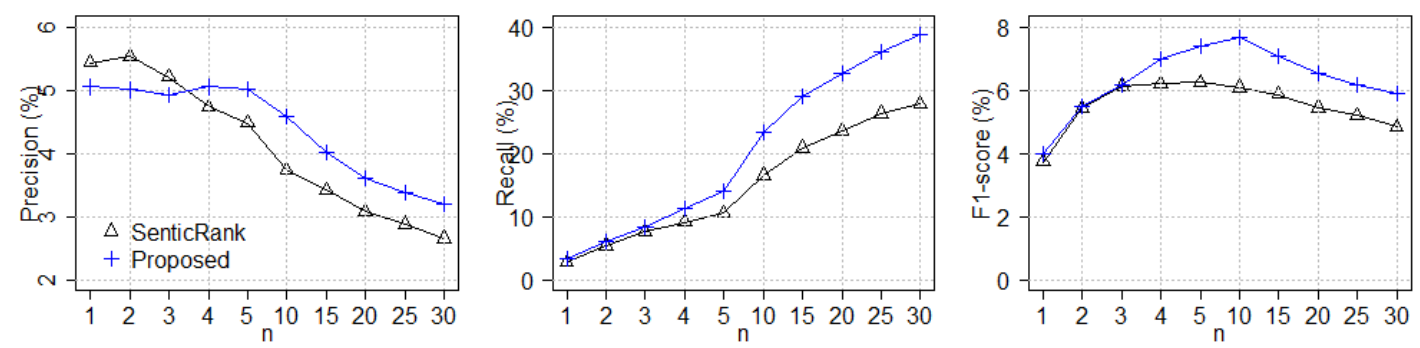

Fig. 6. The comparisons with SenticRank by precision, recall, and f1-score. 
The proposed approach is evaluated against the research based on the tensor factorization: TF and Bayes. Fig. 5 shows the results of the evaluations. All three methods were modeled by the tensor and applied the tensor factorization. The results show that it is important to consider user's feedback rather than using only the existence of the relationship in the tensor modeling, and it is better to consider the history of the user behavior based on the tag for the recommendation. Also, the results describe that it is better to apply BM25 than to use Naïve Bayes technique in profile generation for ranking of recommendation candidates. This implies that the relevance-based profile performs better in item recommendation because BM25 and Naïve Bayes rank documents based on their relevance and preference respectively when a new query comes in [45].

Finally, our approach compared with SenticRank. In [33], the authors suggest two ranking methods using the sentiment for the personalized search in folksonomy: content-based sentiment rank and collaborative sentiment rank. For our experiment, we modified the content-based sentiment rank and excluded the relevance score calculation between query and resources.

$$
\theta\left(u_{i}, r_{a}\right)=e^{\operatorname{Sim}\left(\overrightarrow{u_{l}}, \overrightarrow{r_{a}}\right)+\operatorname{Sim}\left(\overrightarrow{u_{l}^{*}}, \overrightarrow{r_{a}^{*}}\right)}
$$

where Sim is the cosine similarity, $\overrightarrow{u_{l}}$ is tag-based user profile, $\overrightarrow{r_{a}}$ is tag-based resource profile, $\overrightarrow{u_{l}^{*}}$ is sentiment-based user profile, and $\overrightarrow{r_{a}^{*}}$ is sentiment-based resource profile. The tag's polarity is used to obtain sentiment-based profiles. The experiment results are described in Fig. 6. In this result, SenticRank shows lower performance than the proposed method, but it is a better result than our previous study. It implies that generating the tag-based profiles and reflecting the emotions included in user feedback is effective to improve the recommendation quality. The difference in performance between SenticRank and the proposed method appears to be due to differences in the way the emotions are applied. In the SenticRank approach, each tag denoted with a 5-dimensional sentiment vector: pleasantness, attention, sensitivity, aptitude, and polarity.

Our experiments has a limitation that the data used in the experiment is too small. To test the proposed recommendation method, we need data that includes users, items, tag, and ratings all together; however, as the policy for collecting data becomes more rigorous, it is difficult to obtain these data sets. Thus, we used the open data, and only a fraction of the data was available, the actual dataset used in the experiment had to be small. Although the proposed method can be applied if the dataset is large, the speed of the tensor calculation can be affected.

\section{Conclusion}

In our previous study, we suggested a tensor-based tag emotion aware recommendation method for item recommendation in social cataloging services. Modeling the ternary relationships of users, items, and tags as a tensor occurs the problem of data sparsity because the number of the tagged items are small. Also, tensor factorization and reconstruction do not consider the user and item behaviors.

In this paper, we proposed the improved item recommendation method to alleviate the problems. The additional data are generated by item-based collaborative. The expanded data are modeled as a three-order tensor and HOSVD technique is applied for recommending new items. The recommendation candidates are ranked by the previous tagging activity based user 
and item profile. For creating the profiles, we used BM25 algorithm, which is a TF-IDF based ranking method for a document retrieval.

We conducted the experimental evaluation to compare our approach against other recommendation techniques. The results showed that the proposed approach outperform the other recommendation method.

As the future research, to alleviate the problem of increasing the amount of computation as the amount of user data increases, the clustering methods or the parallel methods can be adopted to tensor factorization for reducing the computation time.

\section{References}

[1] L. F. Spiteri, "Social cataloguing sites: features and implications for cataloguing practique and the public library catalogue," in Proc. of Nuevas perspectivas para la difusión y organización del conocimiento: actas del congreso, pp. 769-785, 2009.

[2] J. Buder and C. Schwind, "Learning with personalized recommender systems: A psychological view," Computers in Human Behavior, vol. 28, no. 1, pp. 207-216, 2012. Article (CrossRef Link)

[3] F. O. Isinkaye, Y. O. Folajimi, and B. A. Ojokoh, "Recommendation systems: Principles, methods and evaluation,” Egyptian Informatics Journal, vol. 16, no. 3, pp. 261-273, 2015. Article (CrossRef Link)

[4] J. Bobadilla, F. Ortega, A. Hernando, and A. Gutiérrez, "Recommender systems survey," Knowledge-Based Systems, vol. 46, pp. 109-132, 2013. Article (CrossRef Link)

[5] G. Gonzalez, J. L. De La Rosa, M. Montaner, and S. Delfin, "Embedding emotional context in recommender systems," in Proc. of Data Engineering Workshop, 2007 IEEE 23rd International Conference on, pp. 845-852, 2007. Article (CrossRef Link)

[6] M. Tkalčič, A. Kosir, and J. Tasic, "Affective recommender systems: the role of emotions in recommender systems," in Proc. of The RecSys 2011 Workshop on Human Decision Making in Recommender Systems, pp. 9-13, 2011.

[7] A. K. Milicevic, A. Nanopoulos, and M. Ivanovic, "Social tagging in recommender systems: A survey of the state-of-the-art and possible extensions," Artificial Intelligence Review, vol. 33, no. 3, pp. 187-209, 2010. Article (CrossRef Link)

[8] P. Symeonidis, A. Nanopoulos, and Y. Manolopoulos, "A unified framework for providing recommendations in social tagging systems based on ternary semantic analysis," IEEE Transactions on Knowledge and Data Engineering, vol. 22, no. 2, pp. 179-192, 2010.

Article (CrossRef Link)

[9] N. Ifada and R. Nayak, "Tensor-based Item Recommendation using Probabilistic Ranking in Social Tagging Systems," in Proc. of Www, pp. 805-810, 2014. Article (CrossRef Link)

[10] H. Lim and H.-J. Kim, "Item recommendation using tag emotion in social cataloging services," Expert Systems with Applications, vol. 89, pp. 179-187, 2017. Article (CrossRef Link)

[11] J. Peng, D. D. Zeng, H. Zhao, and F. Wang, "Collaborative filtering in social tagging systems based on joint item-tag recommendations," in Proc. of the 19th ACM international conference on Information and knowledge management, pp. 809-818, 2010. Article (CrossRef Link)

[12] A. Nanopoulos, "Item recommendation in collaborative tagging systems," IEEE Transactions on Systems, Man, and Cybernetics Part A:Systems and Humans, vol. 41, no, 4, pp. 760-771, 2011. Article (CrossRef Link)

[13] K. Sparck Jones, S. Walker, and S. E. Robertson, "A Probabilistic Model of Onformation Retrieval: development and comparative experiments: Part 1," Information Processing \& Management, vol. 63, no. 6, pp. 779-808, 2000. Article (CrossRef Link)

[14] M. Ames and M. Naaman, "Why we tag: motivations for annotation in mobile and online media," in Proc. of the SIGCHI conference on Human factors in computing systems, pp. 971-980, 2007. Article (CrossRef Link)

[15] P. de Meo, E. Ferrara, F. Abel, L. Aroyo, and G.-J. Houben, "Analyzing user behavior across social sharing environments," ACM Transactions on Intelligent Systems and Technology (TIST), 
vol. 5, no. 1, p. 14, 2013. Article (CrossRef Link)

[16] O. Nov and C. Ye, "Why do people tag?: motivations for photo tagging," Communications of the ACM, vol. 53, no. 7, pp. 128-131, 2010. Article (CrossRef Link)

[17] S. Sen, S. K. Lam, A. M. Rashid, D. Cosley, D. Frankowski, J. Osterhouse, F. M. Harper, and J. Riedl, "Tagging, communities, vocabulary, evolution," in Proc. of the 2006 20th anniversary conference on Computer supported cooperative work, pp. 181-190, 2006.

Article (CrossRef Link)

[18] I. Guy, N. Zwerdling, I. Ronen, D. Carmel, and E. Uziel, "Social media recommendation based on people and tags," in Proc. of the 33rd international ACM SIGIR conference on Research and development in information retrieval, pp. 194-201, 2010. Article (CrossRef Link)

[19] Z.-K. Zhang and C. Liu, "Hybrid recommendation algorithm based on two roles of social tags," International Journal of Bifurcation and Chaos, vol. 22, no. 07, p. 1250166, 2012. Article (CrossRef Link)

[20] H.-N. Kim, A. Alkhaldi, A. El Saddik, and G.-S. Jo, "Collaborative user modeling with user-generated tags for social recommender systems," Expert Systems with Applications, vol. 38, no. 7, pp. 8488-8496, 2011. Article (CrossRef Link)

[21] F. Gedikli and D. Jannach, "Rating items by rating tags," in Proc. of the 2010 Workshop on Recommender Systems and the Social Web at ACM RecSys, pp. 25-32, 2010.

[22] F. Gedikli and D. Jannach, "Improving recommendation accuracy based on item-specific tag preferences,” ACM Transactions on Intelligent Systems and Technology (TIST), vol. 4, no. 1, p. 11, 2013. Article (CrossRef Link)

[23] H. Kim and H.-J. Kim, "Improving recommendation based on implicit trust relationships from tags," in Proceedings of the 2nd international conference on computers, networks, systems, and industrial applications, pp. 25-30, 2012.

[24] C. L. Huang, P. H. Yeh, C. W. Lin, and D. C. Wu, "Utilizing user tag-based interests in recommender systems for social resource sharing websites,” Knowledge-Based Systems, vol. 56, pp. 86-96, 2014. Article (CrossRef Link)

[25] L. De Lathauwer, B. De Moor, and J. Vandewalle, “A multilinear singular value decomposition,” SIAM journal on Matrix Analysis and Applications, vol. 21, no. 4, pp. 1253-1278, 2000. Article (CrossRef Link)

[26] P. Symeonidis, A. Nanopoulos, and Y. Manolopoulos, “Tag recommendations based on tensor dimensionality reduction,” in Proc. of the 2008 ACM conference on Recommender systems, pp. 43-50, 2008. Article (CrossRef Link)

[27] Y. Xu, L. Zhang, and W. Liu, "Cubic analysis of social bookmarking for personalized recommendation,” in Proc. of Asia-Pacific Web Conference, pp. 733-738, 2006.

Article (CrossRef Link)

[28] D. Rafailidis and P. Daras, "The TFC model: Tensor factorization and tag clustering for item recommendation in social tagging systems," IEEE Transactions on Systems, Man, and Cybernetics Part A:Systems and Humans, vol. 43, no. 3, pp. 673-688, 2013.

Article (CrossRef Link)

[29] Z. Li and J. Tang, "Weakly supervised deep matrix factorization for social image understanding," IEEE Transactions on Image Processing, vol. 26, no, 1, pp. 276-288, 2017.

Article (CrossRef Link)

[30] Z. Li, J. Tang, and T. Mei, "Deep Collaborative Embedding for Social Image Understanding," IEEE Transactions on Pattern Analysis and Machine Intelligence, vol. 41, no. 9, 2019. Article (CrossRef Link)

[31] Z. Li, J. Tang, and X. He, "Robust structured nonnegative matrix factorization for image representation,” IEEE Transactions on Neural Networks and Learning Systems, vol. 29, no. 5, pp. 1947-1960, 2018. Article (CrossRef Link)

[32] R. Katarya and O. P. Verma, "Recent developments in affective recommender systems," Physica A: Statistical Mechanics and its Applications, vol. 461, pp. 182-190, 2016.

Article (CrossRef Link) 
[33] H. Xie, X. Li, T. Wang, R. Y. K. Lau, T.-L. Wong, L. Chen, F. L. Wang, and Q. Li, "Incorporating sentiment into tag-based user profiles and resource profiles for personalized search in folksonomy," Information Processing \& Management, vol. 52, no. 1, pp. 61-72, 2016. Article (CrossRef Link)

[34] Z. Qingbiao, F. Jie, and G. Xu, "Incorporating sentiment analysis for improved tag-based recommendation," in Proc. of the 2011 IEEE Ninth International Conference on Dependable, Autonomic and Secure Computing, pp. 1222-1227, 2011. Article (CrossRef Link)

[35] R. Dong, M. O’Mahony, Michael P, M. Schaal, K. McCarthy, and B. Smyth, "Combining similarity and sentiment in opinion mining for product recommendation," Journal of Intelligent Information Systems, vol. 46, no. 2, pp. 285-312, 2016. Article (CrossRef Link)

[36] M. Á. García-Cumbreras, A. Montejo-Ráez, and M. C. Díaz-Galiano, "Pessimists and optimists: Improving collaborative filtering through sentiment analysis," Expert Systems with Applications, vol. 40, no. 17, pp. 6758-6765, 2013. Article (CrossRef Link)

[37] J. Sun, G. Wang, X. Cheng, and Y. Fu, "Mining affective text to improve social media item recommendation," Information Processing and Management, vol. 51, no. 4, pp. 444-457, 2015. Article (CrossRef Link)

[38] B. Sarwar, G. Karypis, J. Konstan, and J. Reidl, "Item-based collaborative filtering recommendation algorithms," in Proc. of the tenth international conference on World Wide Web - WWW '01, pp. 285-295, 2001. Article (CrossRef Link)

[39] Y. Koren, R. Bell, and C. Volinsky, "Matrix factorization techniques for recommender systems," Computer, vol. 42, no. 8, 2009. Article (CrossRef Link)

[40] D. Vallet, I. Cantador, and J. M. Jose, "Personalizing web search with folksonomy-based user and document profiles," Lecture Notes in Computer Science (including subseries Lecture Notes in Artificial Intelligence and Lecture Notes in Bioinformatics), pp. 420-431,2010. Article (CrossRef Link)

[41] S. Xu, S. Bao, B. Fei, Z. Su, and Y. Yu, "Exploring folksonomy for personalized search," in Proc. of the 31st annual international ACM SIGIR conference on Research and development in information retrieval - SIGIR '08, pp. 155-162, 2008. Article (CrossRef Link)

[42] V. Batagelj and M. Zaveršnik, "Generalized cores," arXiv preprint cs/0202039, 2002.

[43] E. Cambria, R. Speer, C. Havasi, and A. Hussain, "SenticNet: A Publicly Available Semantic Resource for Opinion Mining," in Proc. of AAAI fall symposium: commonsense knowledge, vol. 10, no. 0, 2010. Article (CrossRef Link)

[44] K. H. L. Tso-Sutter, L. B. Marinho, and L. Schmidt-Thieme, "Tag-aware recommender systems by fusion of collaborative filtering algorithms," in Proc. of the 2008 ACM symposium on Applied computing - SAC '08, pp. 1995-1999, 2008. Article (CrossRef Link)

[45] M. Zhao, H. Li, A. Ratnaparkhi, J. Wang, and H.-W. Hon, "Adapting Document Ranking to Users' Preferences Using Click-Through Data," in Proc. of Asia Information Retrieval Symposium, pp. 26-42, 2006. Article (CrossRef Link) 


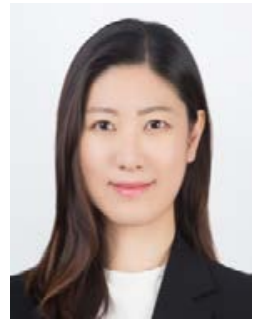

Hyewon Lim received her Ph.D. degree in Computer Science and Engineering from Seoul National University in 2017. Her research interests include recommender system, emotion analysis, natural language processing, information retrieval.

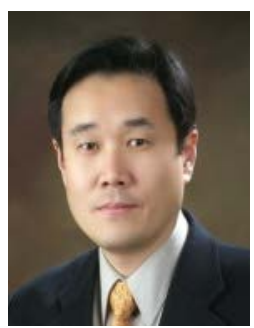

Hyoung-Joo Kim is a Professor in School of Computer Science and Engineering, Seoul National University. He received his M.S. and Ph.D. degree in Computer Science from University of Texas at Austin in 1985 and 1988, respectively. His research interests include database, semantic web, big data, and large-scale data processing 